hängigen Geruchs- nnd Geschmacksprobe noch einige rasch ausführbare Reaktionen zu kennen, die als weitere Kennzeichen bei der Begutachtung zum Vergleiche herangezogen werden können. Voraussichtlich besteht auch die Möglichkeit, die eine oder andere Reaktion bei der Prüfung sonstiger verdorbenen Nahrungsmittel, deren minderwertige Beschaffenheit auf eine Veränderung ihrer Fettsubstanz zurückgeführt werden kann z. B. von Gebäcken, Mebl, Teigwaren, Schokolade u. s. w. in Anwendung zu bringen; auf die Verwendbarkeit der Fuchsinschwefligen Säure zur Erkennung von verdorbenem Mehl wurde schon von A. Zega ${ }^{1}$ ) hingewiesen.

1) Chem.Ztg. 1901, 25, 540.

\title{
Beiträge zur Kenntnis der chemischen Zusammensetzung des Kaffees und der Kaffee-Ersatzstoffe.
}

Von

\section{Prof. Dr. F. Ducháčk in Proßnitz (Mäbren).}

Die Erzengung der Kaffee-Ersatzstoffe erfreut sich in den letzten Jahren eines erheblichen Aufblühens und ist im Gewerbe ein bedeutender Industriezweig geworden. Der Verbrauch steigt ron Jahr zu Jahr, so daf im Handel jetzt schon eine merkliche Nachfrage nach diesen. Surrogaten zu füblen ist, welche auch allein angewendet, einen wohlschmeckenden Absud zu liefern imstande sind.

Weit aber hinter den Fortschritten der Praxis ist die Literatur über die KaffeeErsatzstoffe zurückgeblieben; diese Lücken erkennt man namentlich beim Studium der chemischen Zusammensetzung, welche verhältnismäßig nur sehr wenig Beachtung seitens der Chemiker fand. Größtenteils stützte man sich auf ältere Angaben, welche daher in mancher Richtung nicht mehr der Wirklichkeit entsprechen, da die jetzigen Erzeugnisse durch den inzwischen eingetretenen Fortschritt in der Erzengung von jenen, die seinerzeit dem Handelsverkehre zugeführt wurden, in ihrer Zusammensetzung wesentlich abweichen.

Insbesonders muß hervorgehoben werden, daß man unmöglich die gefundene chemische Zusammensetzung einer Gattung auf alle anderen aus den verschiedenen Fabriken stammenden Kaffee-Ersatzstoffe beziehen kann; denn stimmt die Art und Weise der Erzeugung nicht einmal überein, so können die Erzeugnisse um so weniger eine gleiche chemische Zusammensetzung aufweisen, zumal ja doch gewisse Behandlungen bei der Erzeugung auf dieselbe nicht ohne Einwirkung bleiben. Dazu kommen die mannigfaltigen, gleichfalls unbekannten aromatisehen Zutaten, die, der Mehrzahl nach dem Pflanzenreiche angehörend, zu den fertigen Erzeugnissen in verschiedenen Mengen beigemischt werden und deren Natur dem Geschmacke der Konsumenten angepaßt wird.

In den älteren Arbeiten über die chemische Zusammensetzung der Kaffee-Ersatastoffe finden wir nur eine geringe Anzahl von eingehenden Analysen. Krauch begnügte sich nur mit gleichzeitigen Bestimmungen der löslichen Stoffe und des Zuckers im Kaffee und in den Kaffee-Ersatzstoffen, wogegen Riche und Rémont ihr Hauptaugenmerk auf die Extraktivstoffe, das spezifische Gewicht des Extraktes, die orga- 
nischen Verbindungen und den Stickstoff richteten. Skalweit führte Tabellen an, nach denen man aus dem gefundenen spezifischen Gewichte die Menge des Extraktes aus $100 \mathrm{~g}$ der Substanz berechnen kann. Nach diesen Arbeiten wurden auch die Grundsätze festgelegt, nach welchen man aus der Menge der Extraktivstoffe und des Zuckers die Verfälschungen des Kaffees mit Kaffee-Ersatzstoffen ermitteln kann. Mit emsigem Fleiße verfolgten Swartz, Klencke, Hager und Chevallier die Zusammensetzung der Kaffee-Ersatzstoffe; ausführlichere Analysen finden wir in den Werken von $\mathrm{König,} \mathrm{Petermann,} \mathrm{und} \mathrm{v.} \mathrm{Bibra;} \mathrm{endlich} \mathrm{finden} \mathrm{sich} \mathrm{auch} \mathrm{noch}$ Analysen auf den Etiketten, unter denen die verschiedenen Firmen ihre Surrogate in den Handel bringen. Besonders beachtenswert sind aber die Arbeiten von J. W olff ${ }^{1}$ ), welcher durch die Analyse der frischen, erhitzten und der gerösteten Cichorienwurzel zu folgenden Ergebnissen gelangt ist:

\begin{tabular}{|c|c|c|c|c|c|c|}
\hline \multirow{2}{*}{\multicolumn{2}{|c|}{ Bestandteile }} & & & \multicolumn{3}{|c|}{ Cichorienwurzel } \\
\hline & & & & frisch & erhitzt u, getrocknet & $\overrightarrow{\text { geröstet }}$ \\
\hline Wasser & 5 & . & . & $79,2 \%$ & $17,0^{\circ} \%$ & $16,0 \%$ \\
\hline Asche & & . & . & 1,11 . & - & $2,75 \%$ \\
\hline Zucker. & . . & 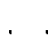 & . & 0,6 & $5,3 \%$ & 14,4 \\
\hline Innlin &.$\quad$. & . & . & $13-15 \%$ & $47-51 \%$ & 9,6 \\
\hline Karamel & . & 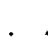 & . & - & - & 9,0 \\
\hline In Wassel & Lösliches & & . & - & - & 61,0 \\
\hline Cellulose &... & & . & 1,29 & - & 9,1 \\
\hline Fett. & . . . . & & . & 0,11 & - & 1,7 \\
\hline Gesamt-st & ickstoff-Sul & bsta & $n z$ & 1,15 & - & 6,15 \\
\hline
\end{tabular}

Die frische und getrocknete Cichorienwurzel enthält beachtenswerte Mengen von Inulin, welches beim Rösten schwindet; indem das Inulin einerseits bei der verhältnismäßig hohen Temperatur des Röstens in dextrinartige Körper, andererseits aber in Karamel übergeführt wird. Der in der gerösteten Cichorie vorhandene Zucker besteht aus einem Gemenge von Glykose und Fruktose, in welchem Fruktose vorherrscht.

In einer anderen Abhandlung befaßt sich J. Wolff ${ }^{2}$ ) mit dem Studium des Inulins. Bei den Analysen der Cichorienwurzel hat er gefunden, daß die Differenzen, die durch Berechnung des Inulins aus der direkten Polarisation einerseits und aus der Drehung der durch Inversion des Inulins erhaltenen Fruktose andererseits erhalten werden, durch einen links drehenden, vergärbaren, dem Inulin ähnlichen Körper veranlaßst werden. Die Bestimmung des nicht vergärbaren und des vergärbaren Inulins erzielte er dadurch, daß er in einem Teile des Extraktes die Menge der durch Inversion erhaltenen Fruktose bestimmte (in der Trockensubstanz der Cichorienwurzel $66^{\circ} \%$ ), in dem anderen Teile des Extraktes aber rief er Alkoholgärung durch Bierhefe hervor, und nach ihrer Beendigung polarisierte er das Filtrat sowohl direkt, als auch nach erfolgter Inversion. Der Unterschied zwischen den Polarisationen (auf Fruktose berechnet) des nicht vergärbaren Inulins einerseits (in der Trockensubstanz der Cichorienwurzel 24,3\%) und der Menge der Fruktose im nicht vergorenen Extrakte andererseits gab das nicht vergärbare Inulin (in der Trockensubstanz 41,7\%

Als Gegenstand meines Studiums wählte ich die Surrogate aus der neuerrichteten Koliner Kaffee-Surrogaten-Fabrik in Proßnitz und zur Vergleichung einen frischen bis zur "kastanienbraunen" Farbe gerösteten 1903-er Ceylon-Kaffee.

1) Ann. chim. analyt. 1899, 4, 157; diese Zeitschrift 1900, 3, 255.

2) Diese Zeitschrift 1900, 3, 593 . 


\section{Untersuchangsverfahren.}

Die Proben für die einzelnen Bestimmungen wurden wie folgt entnommen:

Feigen- und Cichorienkaffee wurden in Originalform genommen, dagegen wurden der echte und der Malzkaffee vor dem Gebrauche gemahlen. Das Abwägen der za allen einzelnen Bestimmungen nötigen Mengen wurde bei Beginn der Analyse aus dem Grunde ausgeführt, weil die zu untersuchenden Stoffe insgesamt und namentlich in Pulverform alle stark hygroskopisch sind.

Zu der A usführung der Analysen sei folgendes bemerkt:

1. Die Feuchtigkeit bezw. die Trockensubstanz wurde durch Trocknen bei $100-105^{\circ} \mathrm{C}$ bis zar Gewichtsbestandigkeit bestimmt.

2. Die Bestimmung des Gesamt-Stickstoffes geschah in der ursprïnglichen Substanz nach der von Maercker abgeänderten Methode von Kjeldahl-Willfarth.

Nach Sisley ${ }^{1}$, Turnbull und Procter ${ }^{2}$ ) habe ich versucht, die Substanz mit Schwefelsäure, Kaliumsulfat and wasserfreiem. Kupfersulfat und auch ohne letzteres - Gunnings-Verfahren, das Gibs on ${ }^{3}$ ) in der neuesten Zeit empfiehlt - zu kochen, fand aber keine besonderen Vorzüge dieser Methoden. Hingegen haben mir die Anleitungen von de $\mathrm{Paepe}$ und $\left.B e a u r a i n s^{*}\right)$ wesentliche Dienste geleistet; dieselben bestimmen durch einen blinden Versuch die in den Reagentien vorhandene Menge Stickstoff, verwerfen die Verwendung des Zinks bei der Destillation und raten an, diesen durch Bimsstein zu ersetzen. Damit die Gesamtmenge des Ammoniaks in die Vorlage gelangt, soll man $2 / 3$ der Flüssigkeit überdestillieren. Hopkins ${ }^{5}$ ) empfiehlt, die sofortige Titration der Säure im Absorptionskolben vorzunehmen, nm dadurch allen möglichen Verlusten von Stickstoff zu begegnen.

Viele Chemiker betrachten die Verwendung von Kaliumsulfid zar Zerstörung des beim Aufschluf unter Zusatz von Quecksilber entstehenden Amidomercurisulfates, als eine unrichtige Maßregel; Maquenne und Roux ${ }^{6}$ ) empfehlen wieder Natriumhypophosphit und Neuber $\mathrm{g}^{7}$ ) erzielt sehr genaue Ergebnisse durch den Gebrauch von Natriumhyposulfit.

Da der Stickstoff-Gehalt der Proteinstoffe der verschiedenen untersuchten Substanzen bis jetzt nicht genau bekannt ist, so wurde zur Umrechnung auf Stickstoff-Substanz stets der allgemein gebräuchliche Faktor 6,25 angewendet.

3. Das Rohfett (Ätherauszug) wurde nach J. König ${ }^{8}$ ) bestimmt. Zu der Extraktion wurde mit Ätzkalk gereinigter Äther genommen, wie ihn Methner empfiehlt, der auf Grund seiner Versuche behauptet, daf die allgemein aufgestellte Bedingung, die Substanz mit alkoholund wasserfreiem Äther auszuziehen nicht eingehalten zu werden brancke. Der im Handel vorkommende Äther ist niemals alkohol- und wasserfrei, wenn er auch als solcher angepriesen wird; er liefert aber trotzdem nur wenig abweiehende Ergebnisse gegenüber absolutem Äther, der über Natrium destilliert wurde. Übrigens ist der Fehler, welcher durch den Gebrauch des nicht ganz reinen Athers begangen wird, sehr umbedeutend gegenüber den Fehlern, die dadurch bedingt werden, daß der Ather einerseits nicht alle in der Substanz enthaltene Fette löst und andererseits aber auch einige Nichtfette zur Lösung bringt.

4. Bei der Auswahl des Verfahrens zur Bestimmung der Rohfaser kommt man in Verlegenheit. Es gibt nur sehr wenige Bestandteile, zu deren Bestimmung eine so grofe

1) Chem.-Ztg. 1899, 28, 671.

2) Chem.-Ztg. 1900, 24, 126.

3) Journ. Amer. chem, Soc. 1904, 96, 105.

4) Bull. de l'Assoc. belge des chimistes 1900, 14, 305 .

5) Jeurn. Amer. chem. Soc. 1898, 20, 961.

$\left.{ }^{6}\right)$ Bull. Sec. chim. 1899, 21, 312.

$\left.{ }^{7}\right)$ Beitr. Chem. Physiol. u. Pathol. 1902, 2, 214.

8) J. K önig, Untersuch. landw. u. gewerbl. wicht. Stoffe. 2. Auf. Berlin 1898, 205.

9) Chem.-Ztg. 1899, 23, 37. 
Anzahl von Verfahren und so mannigfaltiger Art vorgeschlagen sind, wie für diesen. Am meisten werden zwei Verfahron angewendet, von denen das eine darin besteht, daß3 man die Cellulose anszieht und dieselbe quantitativ in der erhaltenen Flüssigkeit bestimmt, während man bei dem anderen Verfahren Lösungsmittel angewendet hat, welche sämtliche Stoffe beseitigen und nur die Cellulose allein unangegriffen zurücklassen. Durch die Hydrolyse der nichtcelluloseartigen Stoffe exhält man aber entweder eine ungenügend gereinigte Cellulose, oder, wenn hierzu kräftige Lösungsmittel angewendet werden, so sind die Ergebnisse der Analyse zu niedrige, der Wirklichkeit nicht entsprechende, weil die Cellulose teilweise angegriffen wird und in Lösung geht. Solche langwierigen und den analytischen Anforderungen nicht entsprechenden Verfahren bilden bei weitem die Mehrzahl; aber eine exakte und bequeme Methode zur Bestimmung der Cellulose ist nicht vorhanden. Die meiste Anwendung fand das von Henneberg und Stohman ${ }^{1}$ ) vorgeschlagene Verfahren, nach welchem der nach je einem halbstündigem Kochen mit 11/4\%-iger Schwefelsäure und 1/4\%-iger Kalilauge verbleibende Rückstand als Rohfaser angesehen wird. Aus den Rahmen der angeführten Ver-

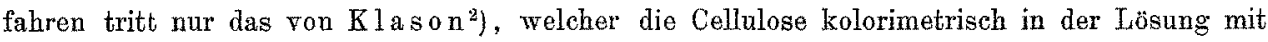
konzentrierter Schwefelsäure bestimmt.

Von den neu veröffentlichten Verfahren hat mir das von J. König ${ }^{3}$ ) besonders zugesagt und dieses habe ich auch zur Bestimmung der Cellulose und des Lignins im Kaffee and den Surrogaten angewendet. Die Hydrolyse der nichtcelluloseartisen Stoffe wurde mit Glycerin und Schwefelsäure nach der Anleitung von König bewerkstelligt; in einem Falle wurde filtriert, gewaschen, getrocknet, gewogen, verascht und neaerdings gewogen (Asche abgezogen) - wodurch Cellulose + Lignin gefunden wurden -; im anderen Falle wurde die Oxydation des Lignins in der Holzfaser mit Wasserstoffsuperoxyd und Ammoniak durchgeführt, die restliche reine Cellulose ausgewaschen, getrocknet, gewogen, verascht und die Asche abgezogen. Die Differenz der beiden Bestimmungen gab sodann das Lignin.

5. Die Pentosane wurden nach dem durch Tollens und Krüger abgeänderten Verfahren von Councler ${ }^{4}$ bestimmt; die Substanz wurde mit Salzsäure von 1,06 spezifischem Gewichte destilliert, in dem Destillate das Furfurol durch Phloroglucin gefällt und das erhaltene Phloroglucid gewogen. Das Zahlenverhältnis der Pentosane gegen die Methylpentosane, die regelmäßige Bestandteile der Pflanzen sind, warde nicht bestimmt. Wichtige Winke für die Bestimmung der Furfuroide geben Andrlik ${ }^{5}$ ), Votoček ${ }^{6}$ ) und Kröber ${ }^{7}$ ) an.

6. Das Koffein wurde in der ursprünglichen Substanz nach $\mathrm{Co}$ maille bestimmt; aus dem Chloroformauszuge wurde nach dem Abdestillieren des Lösungsmittels das Alkaloid in Wasser gelöst und nach dem Abdampfen dieses sodann gewogen.

7. Die in der ursprünglichen Substanz enthaltene Gesamtmenge der anorganischen St offe und ihre vollständige Analyse wurde nach J. König ${ }^{8}$ j durchgeführt. Um den Verlusten an Alkalien vorzubeugen, habe ich die verkohlte Masse mit Wasser ausgelaugt und die so erhaltene Lösung dem weißgebrannten unlöslichen Rückstande zugesetzt. Für die bloßse Aschenbestimmung in den Kaffee-Ersatzstoffen kann das von Kle i ${ }^{9}$ ) vorgeschlagene Verfahren in Anwendung gebracht werden, indem die getrockneten organischen Substanzen unter Zugabe von gebrannter Magnesia verascht werdon. Man kann bei Anwendung einer mäßjigen

1) J. König, Untersuch. landw. u. gewerbl. wicht. Stoffe. 2. Aufl, Berlin 1898, 205.

2) V. Internat. Kongress f. angew. Chemie, Berlin 1908.

3) Diese Zeitschrift 1908, 6, 769.

4) Zeitschr. Rübenzuckerind. 46, 480 .

5) Böhm. Zeitschr. Zuckerind. 1899, 23, 314.

6) Böhm. Zeitschr. Zuckerind. 1899, 24, 229.

7) Journ. Landw. 1901, 48, 357.

8) J. König, Untersuch. landw. u. gewerbl. wicht. Stoffe. 2. Aufl. Berlin 1898, 186.

9) Chem.-Zeitg. 1903, 27, 923. 
Flamme und in kurzer Zeit eine von Kohle vollständig freie Asche erhalten. Neubauer ${ }^{1}$ ) bestimmt die Alkalien direkt in der ursprünglichen Substanz.

8. Die Bestimmung der wasserlöslichen und in Wasser unlöslichen Stoffe geschah nach J Köni ${ }^{2}$ ). Der bei $100-105^{\circ} \mathrm{C}$. bis zum konstanten Gewichte getrosknete, unlösliche Rückstand gab die Gesamtmenge der unlösliehen Stoffe und in denselben wurden bestimmt:

9. Die unlöslichen Mineralstoffe nach dem Absatze 7.

10. Der Stickstoff bzw. die unlösliehen Stickstoffverbindungen nach dem unter 2 beschriebenen Verfahren.

11. Durch Substraktion von Rohfett, Cellulose, Lignin, unlöslichen anorganischen und Stickstoff- Verbindungen von der Gesamtmenge der unlöslichen Stoffe wurde die Menge der sonstigen unloslichen stickstofffreien Verbindungen gewonnen.

12. Der wässerige Auszug aus $25 \mathrm{~g}$ Trockensubstanz wurde in allen Fallen auf $2500 \mathrm{ccm}$ verdünnt und das spezifische Gewicht dieser Lösung mit der Mohr-Westphalschen Wage festgestellt und zwar wurde gefunden:

beim Ceylonkaffee (schmutziggelber Auszug) . . . , 1,0008 g

" Malzkaffee ( " " ") . . . . . 1,0014 "

"Feigenkaffee (rotbrauner ") . . . . . 1,0030"

"Cichorienkaffee ( " , ) . . . 1,0026"

In dem wassserigen Auszuge wurde weiter bestimmt:

13. Durch Abdampfen, Trocknen bis zur Gewichtsbeständigkeit und Veraschen wie unter 7 die Gesamtmenge der wasserlöslichen und die Menge der anorganischen Stoffe,

14. Der Stickstoff bzw. diejm Wasser löslichen Stickstoffrerbindungen wie unter 8 .

15. Das Dextrin wurde von den anderen wasserlöslichen Stoffen mittels Alkohols nach der Anleitnng von J. Königis) getrennt, durch Invertieren mit Salzsäure in Glykose übergeführt und diese nach dem von mir verbesserten Centrifugal-Verfahren von ChapellePellet ${ }^{1}$ ) bestimmt. Wit meiner Arbeit: „Kritische Studien über einige Wägemethoden zur Bestimmung von reduzierenden Zuckern und Vergleichung derselben mit der Centrifugalmethode" ${ }^{5}$ ) habe ich bewiesen, daf diese allereinfachste, am raschesten zum Ziele führende Methode den Verfahren, bei welchen das ausgeschiedene Kupferoxydul mit Asbest in dem Soxhlet'schen Röhrchen oder im Gooch'schen Tiegel aufgefangen wird, nichts nachgibt.

16. Dieses Verfahren wurde auch bei der Bestimmung des reduzierenden Zuckers in dem Auszuge, der nach der Fällung des Dextrins erhalten wird, angewendet. Die gefundene Kupfermenge wurde auf Glykose umgerechnet.

17. Durch Abrechnung des reduzierenden Zuckers, des Dextrins, (des Koffeins), der im Wasser Iöslichen Stickstoffsubstanzen und der anorganischen Stoffe von der Gesamtmenge dex wasserlöslichen Bestandteile wurde die Menge der sonstigen wasserIöslichen stickst offfreien Verbindungen erhalten.

18. Durch Substraktion des Rohfettes, der Cellulose, des Lignins, der Pentosane, des reduzierenden Zuckers, des Dextrins, (des Koffeins), der Gesamtmenge der anorganischen und Stickstoffverbindungen wurden die Angaben für sonstige stickstofffreie $S u b-$ stanzen erhalten.

1) Zeitschr. analyt. Chem. 1904, 43, 14.

2) J. König, Untersuch. landw. u. gewerbl. wicht. Stoffe. 2. Aufl. Berlin 1898, 208.

3) J. König, Untersuch. landw. u. gewerbl. wicht. Stoffe. 2. Aufl. Berlin 1898, 210.

*) Bulletin de l'Assoc. des chimistes de sucrerie et de distillerie 1900, 18, 776.

5) Diese Zeitschrift 1904, 7, 629. 


\section{Ergebnisse der Analysen.}

In der nachstehenden Tabelle I (S. 145) sind die Ergebnisse der Analysen der untersuchten vier Proben, auf ursprüngliche sowie auf Trockensubstanz berechnet, wiedergegeben. Aus diesen Zahlen sind ferner die in Prozenten 1. der in Wasser löslichen Stoffe, 2. der in Wasser unlöslichen Stoffe sowie 3. der Mineralstoffe ausgedrücktem Werte der Tabelle II (S. 146) berechnet.

Die gefundenen Ergebnisse bedürfen keiner weiteren Erörterungen; hervorgehoben mögen nur die auffälligen Unterschiede werden, welche in der chemischen Zusammensetzung des Kaffees und der Kaffee-Ersatzstoffe bestehen und deren man sich mit Vorteil zur Erkennung des einen oder des anderen Produktes bedienen kann.

Der Feigenkaffee und auch der Cichorienkaffee zeicbnen sich durch ihren hohen Gehalt an wasserlöslichen Stoffen aus, welche $3 / 4$ der Trockensubstanz ausmachen; dagegen ergibt der Malzkaffee etwa ${ }^{3}{ }_{i 5}$ unlöslichen Rückstand; als am wenigsten ausgiebig zeigt sich der echte Kaffee, der nur ${ }^{1 / 1}$ seines Gewichtes an wasserlöslichen Stoffen liefert. In bezug auf den Gesamt-Stickstoffgehalt übertrifft der echte Kaffee und der Malzkaffee alle anderen Surrogate $\left({ }^{1 / 10}\right.$ der Trockensubstanz); an wasserlöslicher Stickstoff-Substanz wurde die größte Menge beim echten Kaffee und beim Cichorienkaffee gefunden, dagegen findet sich der höchste Gehalt an unlöslicher Stickstoff-Substanz beim Malzkaffee. Durch den Gehalt an Rohfett ( $1{ }_{6}$ der Trockensubstanz), an Pentosanen ( $1 / 6$ der Trockensubstanz), an Cellulose und an Lignin (fast $1 / 3$ der Trockensubstanz) überragt der echte Kaffee bei weitem die untersuchten Kaffee-Ersatzstoffe.

Reduzierenden Zucker finden wir in großen Mengen beim Feigenkaffee (2/5 der Trockensubstanz) und bein Cichorienkaffee (1/5 der Trockensubstanz), dagegen ist der Gehalt an Zucker im echten Kaffee und im Malzkaffee nur gering. Ein höherer Gehalt an Dextrin ( ${ }^{1 / 10}$ der Trockensubstanz) charakterisiert besonders den Malzkaffee.

In dem Gehalt an Gesamt-Mineralstoften zeigen sich keine nennenswerten Unterschiede; dagegen führt die eingehende Analyse der Asche zu sehr wertvollen Ergebnissen.

Das Kali ist vorherrschend beim echten Kaffee $\left(^{3} / 5\right.$ der Asche), beim Feigenund Cichorienkaffee ( $1 / 2$ der Asche); beim Malzkaffee dagegen erreicht es nicht einmal $1 / 5$ des Aschengewichtes.

Der Malakaffee ist vor dem echten Kaffee und den drei übrigen Kaffee-Ersatzstoffen ausgezeichnet durch seinen Gehalt an Phosphorsäure (1/3 der Asche); an $\mathrm{K}$ ieselsäure enthält neben dem Malzkaffee (1/4 des Aschengewichtes) der Cichorienkaffee die grössten Mengen ( $1 / 7$ der Asche).

Der wichtigste Unterschied liegt aber natürlich in dem Gehalte an Koffein, welcher ja dem echten Kaffee vorwiegend seinen Wert verleiht und ihn von allen gebräuchlichen Ersatzstoffen wesentlich unterscheidet.

Die minder bemittelten Volksklassen, welche sich des verhältnismäßig teueren Kaffees als Genubmittel nicht bedienen können, nehmen vielfach mit dem Absude eines der Kaffee-Ersatzstoffe vorlieb. Für diesen Zweck ist besonders der billige Malzkaffee geeignet, der einen schmackhaften Absud liefert und häufig seines Freiseins von Koffein wegen als diätetisches Mittel empfohlen wird. 
Tabelle I.

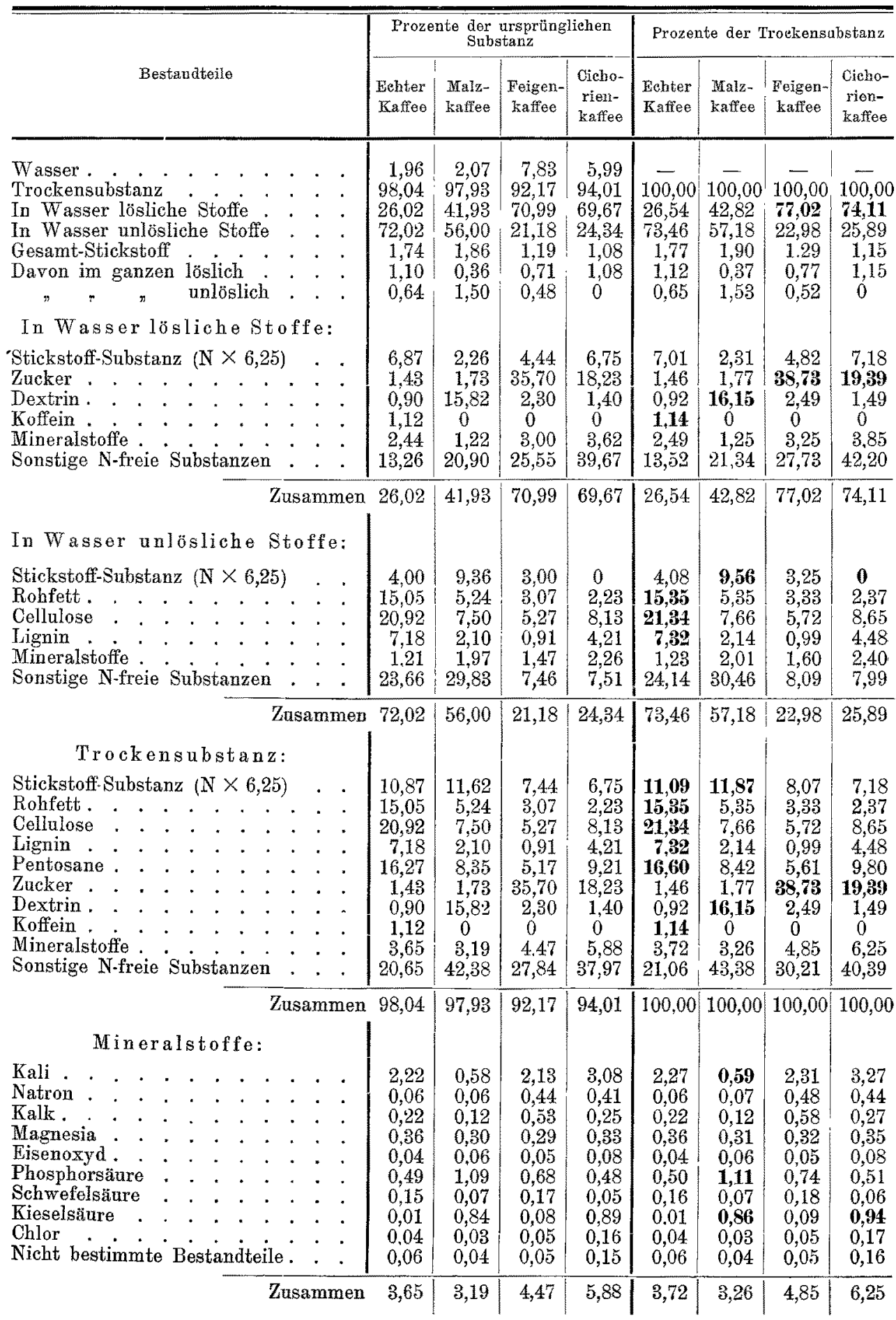


Tabelle II.

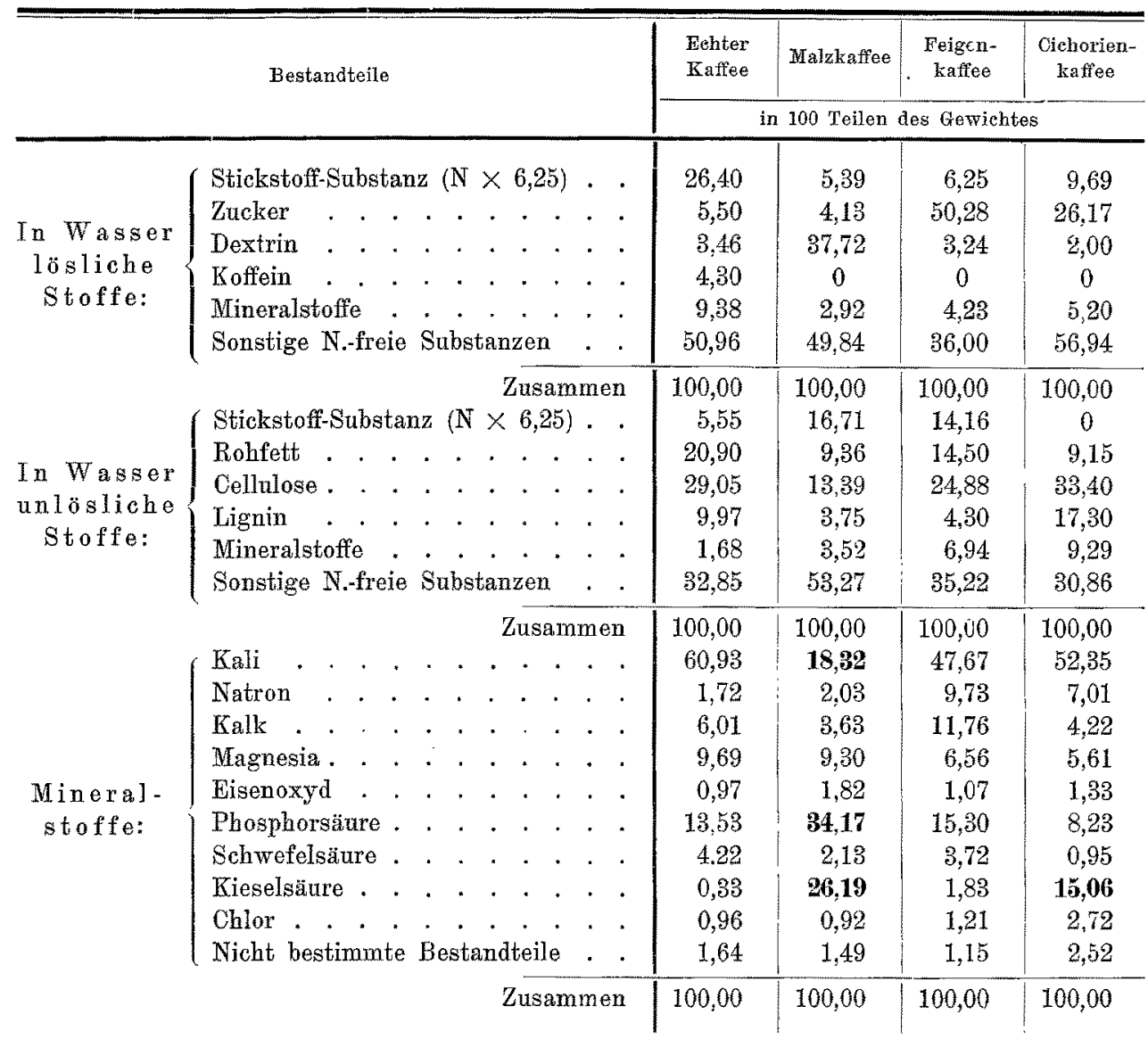

\section{Referate.}

\section{Allgemeine Bestandteile der Nahrungs- und Genufmittel.}

Emil Fischer: Syuthese von Derivaten der Polypeptide. (Berichte Deutsch. chem. Ges. 1903, 36, 2094-2106.) - Nach Ansicht des Verf.'s sind in den Proteinstoffen die Aminosäuren höchstwahrscheinlich nach Art der Säureamide miteinander verkuppelt, wofür nicht allein die Hydrolyse durch Säuren und Alkalien, sondern besonders auch die Beobachtung spricht, dab aus dem Seidenfibroin durch partielle Spaltung ein Stoff entsteht, der wahrscheinlich eine solche Kombination von Glykokoll mit Alanin ist. Verf. ist deshalb schon seit längerer Zeit bemüht gewesen, solche einfache Anhydride der Aminosäuren synthetisch zu bereiten. Während die gewöhnlichen Aminosäuren bisher auf keine Weise in die entsprechenden Säurechloride verwandelt werden konnten, ist dies Verf. gelungen nach Einführung der Karbäthoxylgruppe mit Hilfe von Thionylchlorid. Man kann nach dem Verf, auf diese Weise 